\title{
INFRARED, OPTICAL, AND ULTRAVIOLET OBSERVATIONS OF HYDROGEN LINE EMISSION FROM SEYFERT GALAXIES
}

\author{
J. H. Lacy, ${ }^{1}$ B. T. Solfer, ${ }^{1,2,3}$ G. Neugebauer, ${ }^{2}$ K. Matthews, ${ }^{3}$ AND M. Malkan \\ Division of Physics, Mathematics, and Astronomy, and Palomar Observatory, California Institute of Technology \\ E. E. BeCKLIN \\ Institute for Astronomy, University of Hawaii \\ ChI-Chao Wu ${ }^{1}$ \\ Astronomy Department, Computer Sciences Coporation
}

AND

A. BoGgess ${ }^{1}$ AND T. R. GULL ${ }^{1}$

Laboratory for Astronomy and Solar Physics, Goddard Space Flight Center

Received 1981 September 25; accepted 1981 November 13

\begin{abstract}
Nearly simultaneous observations are reported of $\mathrm{P} \alpha, \mathrm{H} \alpha$, and $\mathrm{H} \beta$ emission from 18 Seyfert galaxies and of Ly $\alpha$ from eight of these galaxies. In many cases, $P \alpha$ is stronger relative to the Balmer lines than is predicted by recombination calculations; reddening appears to be required. Dispersion in the $\mathrm{P} \alpha / \mathrm{H} \alpha / \mathrm{H} \beta$ ratios orthogonal to the reddening track indicates that high densities or optical depths also affect the line flux ratios. Several galaxies, notably NGC 1275 , have very low $\mathrm{P} \alpha / \mathrm{H} \alpha$ ratios. High densities, large optical depths, and reddening probably all contribute to the low observed $\mathrm{L} \alpha / \mathrm{H} \beta$ ratios.
\end{abstract}

Subject headings: galaxies: Seyfert - infrared: sources - ultraviolet: spectra

\section{INTRODUCTION}

It has been known for some time that hydrogen emission-line spectra of active galactic nuclei and quasars do not match the expected recombination spectrum from a plasma ionized by ultraviolet radiation. This discrepancy has led to numerous theoretical attempts to model the line-emitting regions in these objects. Further observational and theoretical studies should help to explain the line-emitting regions and the central objects which excite them.

Balmer line fluxes of Seyfert galaxies have been studied by numerous authors including Anderson (1970), Wampler (1971), Adams and Weedman (1975), Osterbrock (1977), and Koski (1978). Osterbrock studied a sample of 36 Seyfert 1 and broad-line radio galaxies. He found that the Balmer decrement, $\mathrm{H} \alpha / \mathrm{H} \beta / \mathrm{H} \gamma$, could not be explained by a case $\mathrm{B}$ recombination spectrum (see Osterbrock 1974 and $\S$ III, below) reddened by overlying dust. The $\mathrm{H} \alpha / \mathrm{H} \beta$ ratio varied from near the case B value of 2.8 up to more than twiee this value, while $\mathrm{H} \beta / \mathrm{H} \gamma$ was scattered above and below the case $\mathrm{B}$ value of 2.1 and was only weakly correlated with $\mathrm{H} \alpha / \mathrm{H} \beta$. $\mathrm{He}$ suggested that Balmer-line optical depth and

${ }^{1}$ Guest Investigator of the IUE Observatory which is operated by the Goddard Space Flight Center, National Aeronautics and Space Administration.

${ }^{2}$ Visiting Astronomer at the Infrared Telescope Facility which is operated by the University of Hawaii under contract from the National Aeronautics and Space Administration.

${ }^{3}$ Guest Observer at the University of Hawaii, Mauna Kea Observatory. collisional excitation might explain the observations. Koski studied 20 Seyfert 2 and narrow-line radio galaxies, and found that the Balmer-line ratios in these objects could be explained by reddened case B spectra.

The ratio of the Lyman- $\alpha$ flux to Balmer line fluxes was first shown to be unexpectedly low in quasars from a composite spectrum of quasars of different redshifts (Baldwin 1977). This conclusion was confirmed with observations of $L y \alpha$ and Balmer line fluxes from 3C 273 by Davidson, Hartig, and Fastie (1977). Lyman- $\alpha$ fluxes have been measured in a large sample of Seyfert galaxies and low redshift quasars by $\mathrm{Wu}$, Boggess, and Gull (1980). They find that $\mathrm{Ly} \alpha / \mathrm{H} \beta$ varies from $\sim 2$ to 20 , whereas simple photoionization calculations predict $\mathrm{Ly} \alpha / \mathrm{H} \beta \sim 40$. When dereddened by an amount derived from the observed $\mathrm{H} \alpha / \mathrm{H} \beta$ ratio, $\mathrm{Ly} \alpha / \mathrm{H} \beta$ varies from $\sim 4$ to more than 40 , confirming the conclusion from optical observations that reddening alone cannot explain the anomalous hydrogen line spectra of these objects.

An observation of extragalactic $\mathrm{P} \alpha$ emission, from 3C 273, was first published by Grasdalen (1976). This line is particularly useful because $\mathrm{P} \alpha$ and $\mathrm{H} \beta$ arise from the same upper level and so their ratio is insensitive to collisional excitation and deexcitation. Neugebauer $e t$ al. (1980) made infrared, optical, and ultraviolet observations of the Seyfert 2 galaxy NGC 1068. They found that reddening of $E_{B-V}=0.4$ mag could explain the deviations of $\mathrm{P} \alpha, \mathrm{H} \alpha, \mathrm{H} \beta$, and $\mathrm{Ly} \alpha$, from case B ratios and explain the observed [S II] and $\mathrm{He}$ II line ratios. The four lines $P \alpha, H \alpha, H \beta$, and $L y \alpha$ have also been observed in quasars by Soifer et al. (1981) and Puetter 
et al. (1981). In many of these objects, $\mathrm{P} \alpha / \mathrm{H} \beta$ is near the high density case $\mathrm{B}$ value, 0.28 , whereas $\mathrm{H} \alpha / \mathrm{H} \beta$ is typically larger and $\mathrm{Ly} \alpha / \mathrm{H} \beta$ typically smaller than expected in a recombining plasma.

In this paper, observations of $\mathrm{P} \alpha, \mathrm{H} \alpha$, and $\mathrm{H} \beta$ from 14 Seyfert 1 and broad line radio galaxies, two intermediate, and two Seyfert 2 galaxies are reported, as well as observations of $\mathrm{Ly} \alpha$ from eight of these galaxies. In most cases the various observations of each galaxy were coincident within about a month. These observations are used to discuss the line emitting regions in Seyfert galaxies and to compare these objects with quasars.

\section{OBSERVATIONS}

Infrared observations were made on several telescopes. The 1979 May observations were made at the University of Hawaii Mauna Kea Observatory $2.2 \mathrm{~m}$ telescope with a $\Delta \lambda / \lambda=0.013$ circular variable filter spectrometer with an InSb detector. An 8" diameter focal plane aperture was used. The 1979 December observations were made at the NASA Infrared Telescope Facility $3 \mathrm{~m}$ telescope on Mauna Kea with the same spectrometer, using a $6^{\prime \prime}$ diameter aperture. Even at the $4300 \mathrm{~m}$ elevation of Mauna Kea, the transmission of the Earth's atmosphere is severly reduced by water vapor absorption near the wavelengths of the redshifted $\mathrm{P} \alpha$ lines. The observations of the galaxies at each wavelength were hence alternated with observations of nearby early-type stars. Line fluxes were obtained from the data by assuming the flux densities of the comparison stars follow a power law in frequency and then comparing the observed ratios of object signal to stellar signal with calculated ratios of model emission-line profiles and power-law stellar spectra, each of which was convolved with atmospheric transmission and the instrumental profile. The reduction was the same as applied to NGC 1068 by Neugebauer et al. (1980), and further details are given therein.

Two of the observed galaxies, 3C 227 and III Zw 2, have redshifts large enough to shift $\mathrm{P} \alpha$ into the relatively clear $2 \mu \mathrm{m}$ atmospheric window. They were observed with the $2.5 \mathrm{~m}$ Hooker Telescope on Mount Wilson. ${ }^{4}$ The spectrometer used in Hawaii was used for these observations with a $20^{\prime \prime}$ focal plane aperture.

The 1980 infrared observations were made with the NASA Kuiper Airborne Observatory $0.9 \mathrm{~m}$ telescope, flying at an altitude of $12.5 \mathrm{~km}$, to avoid the uncertainties in the correction for absorption by water vapor in the Earth's atmosphere. A circular variable filter spectrometer with a resolution of $\Delta \lambda / \lambda=0.05$ and an InSb detector was used. The focal plane aperture diameter was $28^{\prime \prime}$.

Balmer line observations were made in collaboration with W. L. W. Sargent, A. G. de Bruyn, A. C. S. Readhead, and J. B. Oke with the multichannel spectrophotometer on the $5 \mathrm{~m}$ Hale telescope. A $10^{\prime \prime}$ diameter

\footnotetext{
${ }^{4}$ Observations were made at Mount Wilson Observatory, Carnegie Institution of Washington, under a cooperative agreement with the California Institute of Technology.
}

focal plane aperture was used. Below $5700 \AA$ the spectral resolution was $40 \AA$; at longer wavelengths it was $80 \AA$. The uncertainty in the optical line fluxes is dominated by the difficulty in determining the continuum. The problem is particularly bad for $\mathbf{H} \beta$ in Mrk 231 which has very strong Fe II bands. An additional smaller uncertainty resulted from the correction for [N II] $\lambda \lambda 6548$, 6583 contamination of $\mathrm{H} \alpha \lambda 6563$. Since these lines were not resolved, the [N $\mathrm{NI}]$ flux was estimated by measuring the $[\mathrm{O}$ III $] \lambda \lambda 4959,5007$ flux, and assuming [N II $] /[\mathrm{O}$ III] to be equal to published values for each galaxy, taken mostly from Osterbrock (1977). In cases where $[\mathrm{N} \mathrm{II}] /[\mathrm{O}$ III $]$ was not available, a ratio of $0.2 \pm 0.2$, which is typical of the galaxies observed by Osterbrock, was used. We chose not to try to measure $\mathrm{H} \gamma$ fluxes from the multichannel spectra, because of the difficulty in determining the continuum near $\mathbf{H} \gamma$ in the presence of broad Fe II features.

The Ly $\alpha$ observations were made with the International Ultraviolet Explorer (IUE) satellite (Boggess et al. $1978 a, b)$. The short wavelength prime (SWP) camera was used in the low dispersion mode with a resolution of $\sim 5 \AA$. The flux calibration of Bohlin and Holm (1980) was used. In most cases the $10^{\prime \prime} \times 20^{\prime \prime}$ focal plane aperture was used, although it was necessary when observing NGC 1068 and NGC 4151 to use the $3^{\prime \prime}$ diameter aperture to separate the geocoronal $\mathrm{Ly} \alpha$ emission from that of the galaxies. In these cases the Ly $\alpha$ equivalent width was measured with the small aperture, and the continuum flux was measured with the large aperture. The Ly $\alpha$ flux was then determined by assuming the equivalent width to be independent of aperture. Including pointing, statistical, and calibration uncertainties, the uncertainties in the Ly $\alpha$ fluxes are approximately $\pm 20 \%$.

\section{RESULTS}

The observation dates and measured line fluxes, including estimated [ $\mathrm{N}$ II] fluxes used to correct for contamination of $\mathrm{H} \alpha$, are given in Table 1 . Uncertainties, which are listed under the line fluxes, include $1 \sigma$ statistical and estimated maximum systematic uncertainties. Line flux ratios and other derived quantities are given in Table 2.

Throughout this paper, the Menzel and Baker case B radiative recombination cascade line flux ratios (see Osterbrock 1974) are used as a convenient and familiar basis for comparison, although the densities and optical depths of the broad line emitting regions of Seyfert galaxies are thought to be large enough to invalidate the case $\mathrm{B}$ assumptions. The adopted case $\mathrm{B}$ ratios are $\mathrm{P} \alpha: \mathrm{H} \alpha: \mathrm{H} \beta: \mathrm{Ly} \alpha=0.28: 2.8: 1: 35$. The $\mathrm{P} \alpha: \mathrm{H} \alpha: \mathrm{H} \beta$ ratios are for a radiative cascade in a $10^{4} \mathrm{~K}$ plasma assuming statistical population of the angular momentum sublevels of hydrogen (see Seaton 1959). For Ly $\alpha$, the amount of collisional excitation expected in a $10^{4} \mathrm{~K}$ plasma was included (see Krolik and McKee 1978). For the purpose of discussing reddening, extinctions at the wavelengths of these lines are taken to be $A_{\mathrm{P} \alpha}=0.37 \mathrm{mag}$, $A_{\mathrm{H} \alpha}=2.52 \mathrm{mag}, A_{\mathrm{H} \beta}=3.65 \mathrm{mag}$, and $A_{\mathrm{Ly} \alpha}=10.07 \mathrm{mag}$ for $E_{B-V}=1$ mag (Savage and Mathis 1979). In using 
TABLE 1

LiNE FLuXES

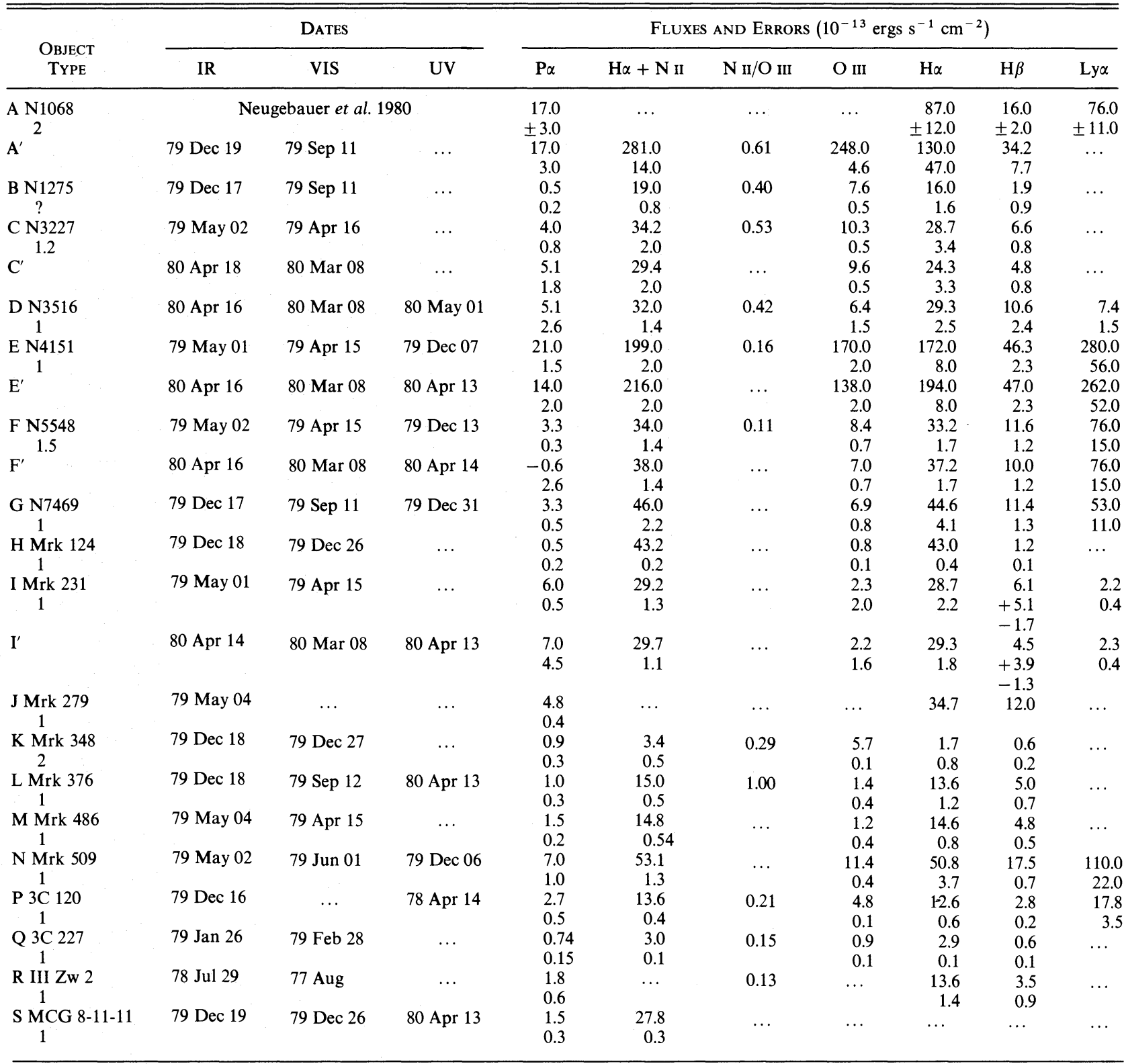

a standard extinction curve it is assumed that the intervening dust lies far enough from the nuclei of the galaxies that light is not scattered into the beam, so that both absorption and scattering contribute to the extinction.

The observed $\mathrm{P} \alpha / \mathrm{H} \alpha$ and $\mathrm{H} \alpha / \mathbf{H} \beta$ flux ratios are plotted in Figure 1. No differences are obvious in this plot between Seyfert 1 and Seyfert 2 galaxies, although too few Seyfert 2 galaxies were observed to allow a statistically significant comparison. The $\mathrm{P} \alpha / \mathrm{H} \alpha$ and $\mathrm{H} \alpha / \mathrm{H} \beta$ ratios of quasars observed by Soifer et al. (1981) and Puetter et al. (1981) are shown with the Seyfert galaxy ratios in Figure 2. Although the uncertainties are large, it appears that the $\mathrm{P} \alpha / \mathrm{H} \alpha / \mathrm{H} \beta$ flux ratios of essentially all active galactic nuclei fall near either the reddened case $\mathrm{B}$ line or the $\mathrm{P} \alpha / \mathrm{H} \beta=0.28$ line. Seyfert galaxies tend to belong to the first group whereas quasars tend to belong to the second, although there is a substantial overlap between Seyferts and quasars, and many of both types of objects have line ratios close to the case B ratios. There is a tendency for the Seyfert points to lie below the reddening track, but Seyfert galaxies with $\mathrm{P} \alpha / \mathrm{H} \alpha / \mathrm{H} \beta$ far from both the reddening and $\mathrm{P} \alpha / \mathrm{H} \beta=0.28$ tracks are rare. 
TABLE 2

Line Flux Ratios

\begin{tabular}{|c|c|c|c|c|c|c|c|c|c|}
\hline \multirow{2}{*}{$\begin{array}{c}\text { OBJECT } \\
\text { TYPE }\end{array}$} & \multicolumn{4}{|c|}{$F_{l} / F_{\mathbf{H} \beta}$} & \multicolumn{3}{|c|}{$\left[F_{l} / F_{\mathbf{H} \beta}\right] /\left[F_{l} / F_{\mathbf{H} \beta}\right]$} & \multirow[b]{2}{*}{$E_{B-V}$} & \multirow{2}{*}{$\begin{array}{l}\text { DEREDDENED } \\
\text { Ly } \alpha / \mathbf{H} \beta\end{array}$} \\
\hline & $\mathrm{P} \alpha$ & $\mathrm{H} \alpha$ & $\mathbf{H} \beta$ & Ly $\alpha$ & $\mathrm{P} \alpha$ & $\mathrm{H} \alpha$ & Ly $\alpha$ & & \\
\hline A N1068 & 1.06 & 5.44 & $\begin{array}{r}1.00 \\
\end{array}$ & 4.75 & 3.79 & 1.94 & 0.14 & 0.44 & $64.6^{\mathrm{a}}$ \\
\hline 2 & \pm 0.19 & \pm 0.75 & \pm 0.13 & \pm 0.72 & \pm 0.67 & \pm 0.27 & \pm 0.02 & \pm 0.07 & \\
\hline \multirow[t]{2}{*}{$\mathbf{A}^{\prime}$} & 0.50 & 3.80 & 1.00 & 1.79 & $\ldots$ & 1.35 & $\ldots$ & 0.20 & $\ldots$ \\
\hline & 0.20 & 1.37 & 0.23 & 0.71 & & 0.49 & & 0.15 & \\
\hline B N1275 & 0.26 & 8.42 & 1.00 & $\ldots$ & 0.94 & 3.00 & $\ldots$ & -0.02 & $\ldots$ \\
\hline$?$ & 0.11 & 0.84 & 0.47 & & 0.38 & 0.30 & & 0.21 & \\
\hline C N3227 & 0.61 & 4.35 & 1.00 & $\ldots$ & 2.16 & 1.55 & $\ldots$ & 0.26 & $\ldots$ \\
\hline 1.2 & 0.12 & 0.52 & 0.12 & & 0.43 & 0.18 & & 0.08 & \\
\hline \multirow{2}{*}{$\mathrm{C}^{\prime}$} & 1.06 & 5.06 & 1.00 & $\ldots$ & 3.79 & 1.80 & $\ldots$ & 0.44 & $\ldots$ \\
\hline & 0.38 & 0.69 & 0.17 & & 1.34 & 0.24 & & 0.13 & \\
\hline D N3516 & 0.48 & 2.76 & 1.00 & 0.70 & 1.72 & 0.98 & 0.020 & 0.18 & 2.0 \\
\hline 1 & 0.25 & 0.24 & 0.23 & 0.14 & 0.88 & 0.08 & 0.004 & 0.18 & \\
\hline E N4151 & 0.45 & 3.71 & 1.00 & 6.05 & 1.62 & 1.32 & 0.17 & 0.16 & 15.5 \\
\hline 1 & 0.03 & 0.17 & 0.05 & 1.21 & 0.12 & 0.06 & 0.03 & 0.03 & \\
\hline \multirow[t]{2}{*}{$\mathrm{E}^{\prime}$} & 0.30 & 4.13 & 1.00 & 5.57 & 1.06 & 1.47 & 0.16 & 0.02 & 6.3 \\
\hline & 0.04 & 0.17 & 0.05 & 1.11 & 0.15 & 0.06 & 0.03 & 0.05 & \\
\hline F N5548 & 0.28 & 2.86 & 1.00 & 6.55 & 1.02 & 1.02 & 0.19 & 0.01 & 6.8 \\
\hline 1.5 & 0.03 & 0.15 & 0.10 & 1.31 & 0.09 & 0.05 & 0.04 & 0.05 & \\
\hline \multirow{2}{*}{$F^{\prime}$} & -0.06 & 3.72 & 1.00 & 7.60 & -0.21 & 1.32 & 0.22 & $\ldots$ & $\ldots$ \\
\hline & 0.26 & 0.17 & 0.12 & 1.52 & 0.93 & 0.06 & 0.04 & & \\
\hline G N7469 & 0.29 & 3.91 & 1.00 & 4.65 & 1.03 & 1.39 & 0.13 & 0.01 & 5.0 \\
\hline 1 & 0.04 & 0.36 & 0.11 & 0.93 & 0.16 & 0.13 & 0.03 & 0.06 & \\
\hline H Mrk 124 & 0.42 & 3.42 & 1.00 & $\ldots$ & 1.49 & 1.22 & $\ldots$ & 0.13 & $\ldots$ \\
\hline 1 & 0.17 & 0.33 & 0.08 & & 0.60 & 0.12 & & 0.14 & \\
\hline I Mrk 231 & 0.98 & 4.70 & 1.00 & 0.36 & 3.51 & 1.67 & 0.010 & 0.42 & 4.2 \\
\hline 1 & 0.08 & 0.36 & 0.56 & 0.07 & 0.29 & 0.13 & 0.002 & 0.19 & \\
\hline \multirow[t]{2}{*}{$\mathbf{I}^{\prime}$} & 1.56 & 6.51 & 1.00 & 0.51 & 5.56 & 2.32 & 0.014 & 0.57 & 14.7 \\
\hline & 1.00 & 0.40 & 0.58 & 0.09 & 3.57 & 0.14 & 0.003 & 0.29 & \\
\hline J Mrk 279 & 0.40 & 2.89 & 1.00 & $\ldots$ & 1.43 & 1.03 & $\ldots$ & 0.12 & $\ldots$ \\
\hline 1 & 0.03 & & & & 0.12 & & & 0.03 & \\
\hline K Mrk 348 & 1.50 & 2.83 & 1.00 & $\ldots$ & 5.36 & 1.01 & $\ldots$ & 0.56 & $\ldots$ \\
\hline 2 & 0.50 & 1.33 & 0.33 & & 1.79 & 0.47 & & 0.16 & \\
\hline L Mrk 376 & 0.20 & 2.72 & 1.00 & $\ldots$ & 0.71 & 0.97 & $\ldots$ & -0.11 & $\ldots$ \\
\hline 1 & 0.06 & 0.24 & 0.14 & & 0.21 & 0.09 & & 0.11 & \\
\hline M Mrk 486 & 0.31 & 3.04 & 1.00 & $\ldots$ & 1.12 & 1.08 & $\ldots$ & 0.04 & $\ldots$ \\
\hline 1 & 0.04 & 0.17 & 0.10 & & 0.15 & 0.06 & & 0.06 & \\
\hline N Mrk 509 & 0.40 & 2.90 & 1.00 & 6.29 & 1.43 & 1.03 & 0.18 & 0.12 & 12.6 \\
\hline 1 & 0.06 & 0.21 & 0.04 & 1.26 & 0.20 & 0.08 & 0.04 & 0.05 & 4.5 \\
\hline P 3C 120 & 0.96 & 4.50 & 1.00 & 6.36 & 3.44 & 1.60 & 0.18 & 0.41 & 71.5 \\
\hline 1 & 0.18 & 0.21 & 0.07 & 1.29 & 0.64 & 0.08 & 0.04 & 0.07 & 31.8 \\
\hline Q 3C 227 & 1.23 & 4.83 & 1.00 & $\ldots$ & 4.40 & 1.72 & $\ldots$ & 0.49 & $\ldots$ \\
\hline 1 & 0.25 & 0.17 & 0.17 & & 0.89 & 0.06 & & 0.09 & \\
\hline R III Zw 2 & 0.51 & 3.89 & 1.00 & $\ldots$ & 1.84 & 1.38 & $\ldots$ & 0.20 & $\ldots$ \\
\hline 1 & 0.17 & 0.40 & 0.26 & & 0.61 & 0.14 & & 0.14 & \\
\hline S MCG 8-11-11 & 0.31 & 5.24 & 1.00 & $\ldots$ & 1.09 & 1.87 & $\ldots$ & 0.03 & $\ldots$ \\
\hline 1 & 0.06 & 0.73 & 0.10 & & 0.22 & 0.26 & & 0.07 & \\
\hline
\end{tabular}

${ }^{a}$ The uncertainties in the dereddened $\mathrm{Ly} \alpha / \mathrm{H} \beta$ values are large and are dominated by the uncertainties in the reddening correction, which assumes $E_{\mathrm{L} y \alpha-\mathrm{H} \beta}=6.4 E_{B-V}$.

The $\mathrm{P} \alpha, \mathrm{H} \alpha, \mathrm{H} \beta$, and $\mathrm{Ly} \alpha$ line fluxes of the galaxies in which all four lines were observed are displayed in Figure 3. Galaxies on the reddened case B track in Figures 1 and 2 have $P \alpha, H \alpha$, and $H \beta$ points on a straight line in Figure 3 with a slope proportional to the reddening. Those on the $\mathrm{P} \alpha / \mathrm{H} \beta=0.28$ track form an inverted $V$ here. The observed $\mathrm{Ly} \alpha / \mathrm{H} \beta$ ratios are all below the adopted case B ratio of 35. The $\mathrm{Ly} \alpha / \mathrm{H} \beta$ ratios, when dereddened assuming $\mathrm{P} \alpha / \mathrm{H} \beta=0.28$, vary from $\sim 2$ to $\sim 40$ (see Table 2). Within their uncertainties, the fluxes of all four lines from NGC 1068, 3C 120, and possibly Mrk 231 fall on reddening lines in Figure 3.
For NGC 4151 and NGC 7469, H $\alpha, \mathrm{H} \beta$, and Ly $\alpha$ fall on a single line, although $\mathbf{P} \alpha / \mathbf{H} \beta$ indicates considerably less reddening than would be required to explain the $\mathrm{H} \alpha / \mathrm{H} \beta$ and $\mathrm{H} \beta / \mathrm{Ly} \alpha$ ratios.

Five galaxies have been observed twice. The two measurements for Mrk 231 (labeled I and I' in Fig. 1 and the tables), for NGC 3227 (C), and for NGC 5548 (F) are not significantly different. A significant $(3.5 \sigma)$ decrease in $\mathrm{P} \alpha$ was seen in NGC 4151 (E), however. The P $\alpha$ flux of NGC 4151 observed on 1980 April 16 was roughly two-thirds that observed a year earlier on 1979 May 1. The 1980 observations were made during a period 


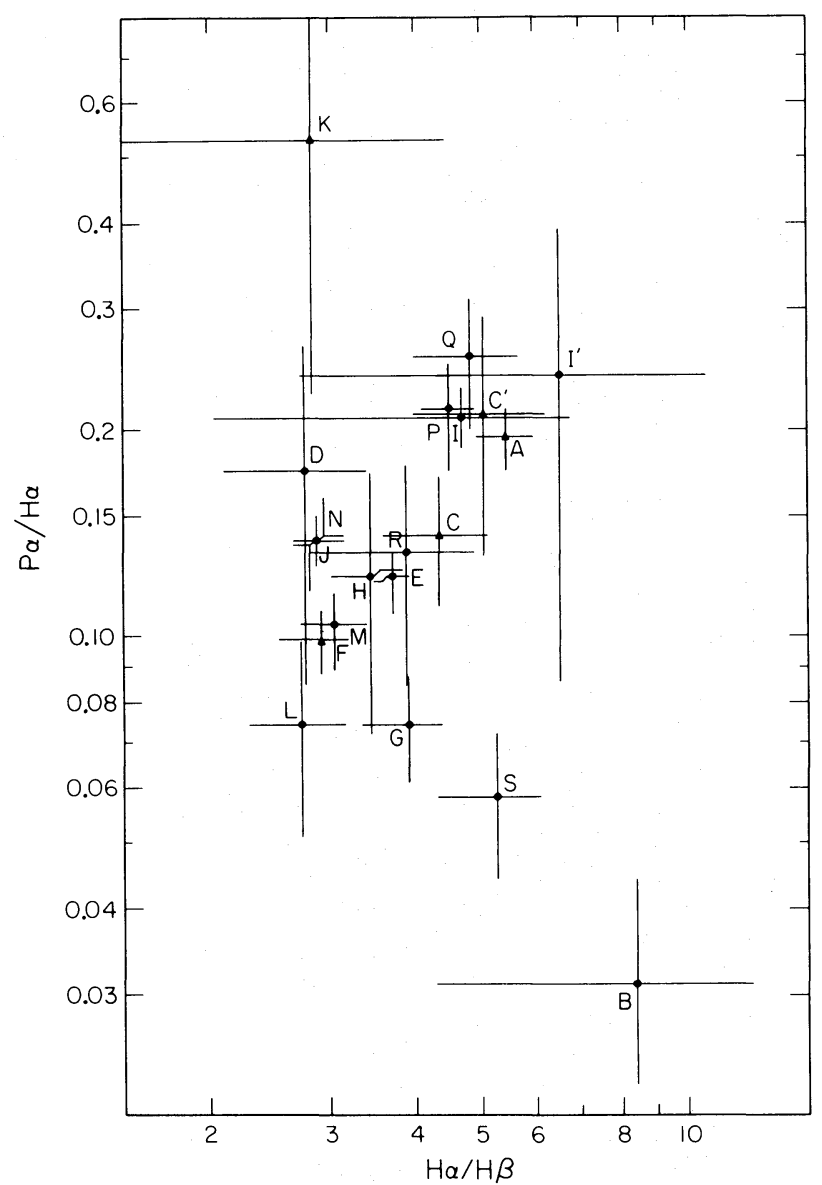

Fig. 1

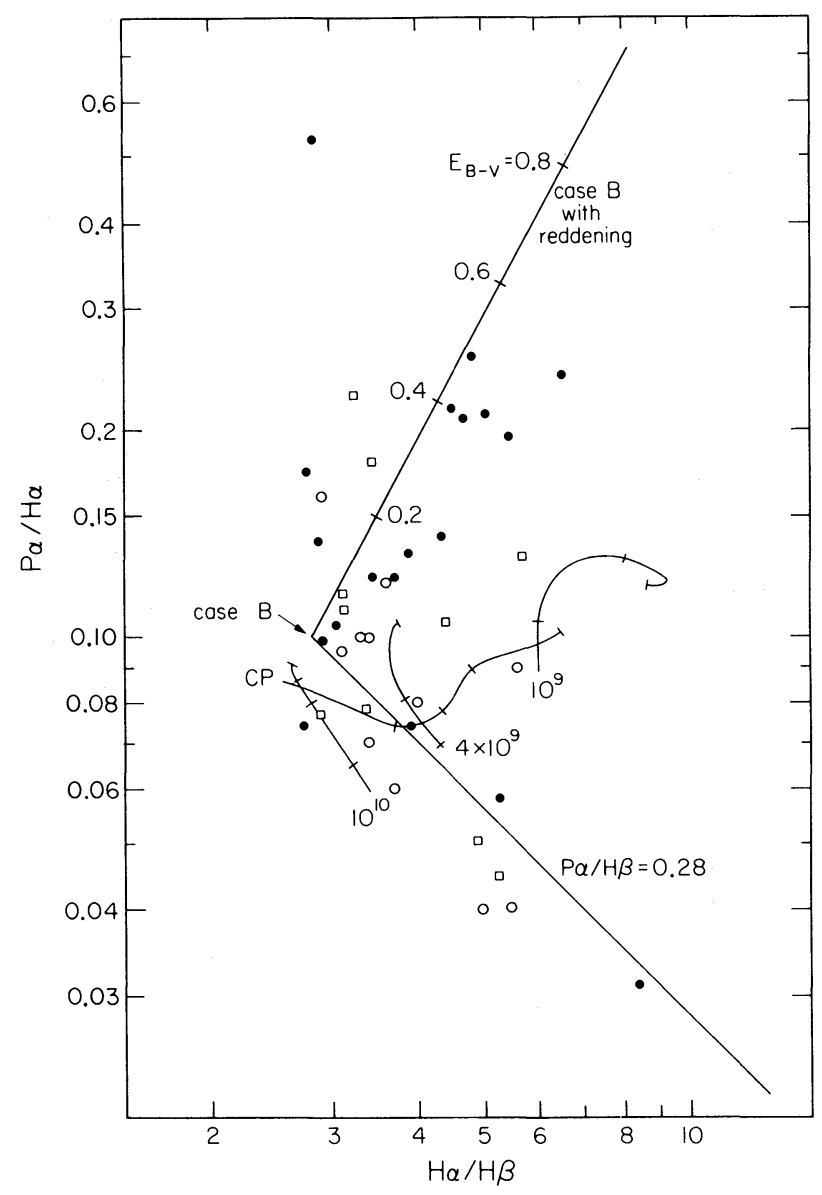

FIG. 2

Fig. 1.-Observed $\mathrm{P} \alpha / \mathrm{H} \alpha$ and $\mathrm{H} \alpha / \mathrm{H} \beta$ line flux ratios from Seyfert galaxies. Circles indicate type 1 Seyfert galaxies and broad line radio galaxies; triangles indicate type 2 and intermediate galaxies. Error bars represent $1 \sigma$ statistical uncertainties plus estimated maximum systematic errors. Optical errors are dominated by the uncertainties in the determination of the continuum. The galaxies shown are: $A$, NGC 1068; $B$, NGC $1275 ; C$, NGC $3227 ; D$, NGC $3516 ; E$, NGC $4151 ; F$, NGC 5548; $G$, NGC 7469; $H$, Mrk 124; $I$, Mrk 231; $J$, Mrk 279; K, Mrk 348; $L$, Mrk 376; M, Mrk 486; $N$, Mrk 509; $P$, 3C 120; Q, 3C 227; R, III Zz 2; $S$, MCG 8-11-11. Primed letters indicate the second observation of a galaxy.

FIG. 2.-Line flux ratios in Seyfert galaxies and quasars. Solid circles represent Seyfert galaxies, open circles are quasars from Soifer et al. (1981), and open squares are quasars from Puetter et al. (1981). The two diagonal lines which meet at the case B point show the expected ratios for a case B spectrum reddened by varying amounts of dust external to the line-emitting region (upper line), and for $\mathbf{P} \alpha / \mathrm{H} \beta=0.28$, the case $\mathrm{B}$ ratio, with $\mathrm{H} \alpha / \mathrm{H} \beta>2.8$ (lower line). The curves show the dependence of the line ratios on optical depth as calculated by Kwan and Krolik (1981), labeled at the high optical depth end by the gas densities, $N_{0}$, and as calculated by Canfield and Puetter (1981) for $N_{0}=10^{10} \mathrm{~cm}^{-3}$, labeled CP. Each curve starts at a Lyman edge optical depth of $\tau_{\mathrm{L}}=10^{3}$ and has tick marks at $\tau_{\mathrm{L}}=10^{3}, 10^{4}, 10^{5}$, and in some cases $10^{6}$.

in which the ultraviolet continuum and $\mathrm{C}$ IV line fluxes were observed to vary. These fluxes decreased by a factor of 1.5 between April 1 and April 21 (M.-H. Ulrich, private communication). In contrast, the 1980 visual observations, made on March 8, and the Ly $\alpha$ observations on April 13 were unchanged from 1979. The data are thus consistent with either an abrupt change in all line fluxes from 1980 April 13 to April 16 or nonsimultaneous variation of the different lines. Because these changes occurred during the period in which the measurements of the various hydrogen line fluxes were made, the 1980 observations are not included in the discussion of line flux ratios below.

NGC 1275, with the largest Balmer decrement and smallest $\mathrm{P} \alpha / \mathrm{H} \alpha$ ratio, occupies a unique position in Figures 1 and 2 . Interestingly, it is also the only object observed which exhibits large rapid (night to night) photometric variability and strong variable linear polarization. In addition, its morphology is very different from that of other Seyfert galaxies. It appears to be a disturbed elliptical galaxy, whereas Seyfert galaxies are almost always spirals (Adams 1977). 


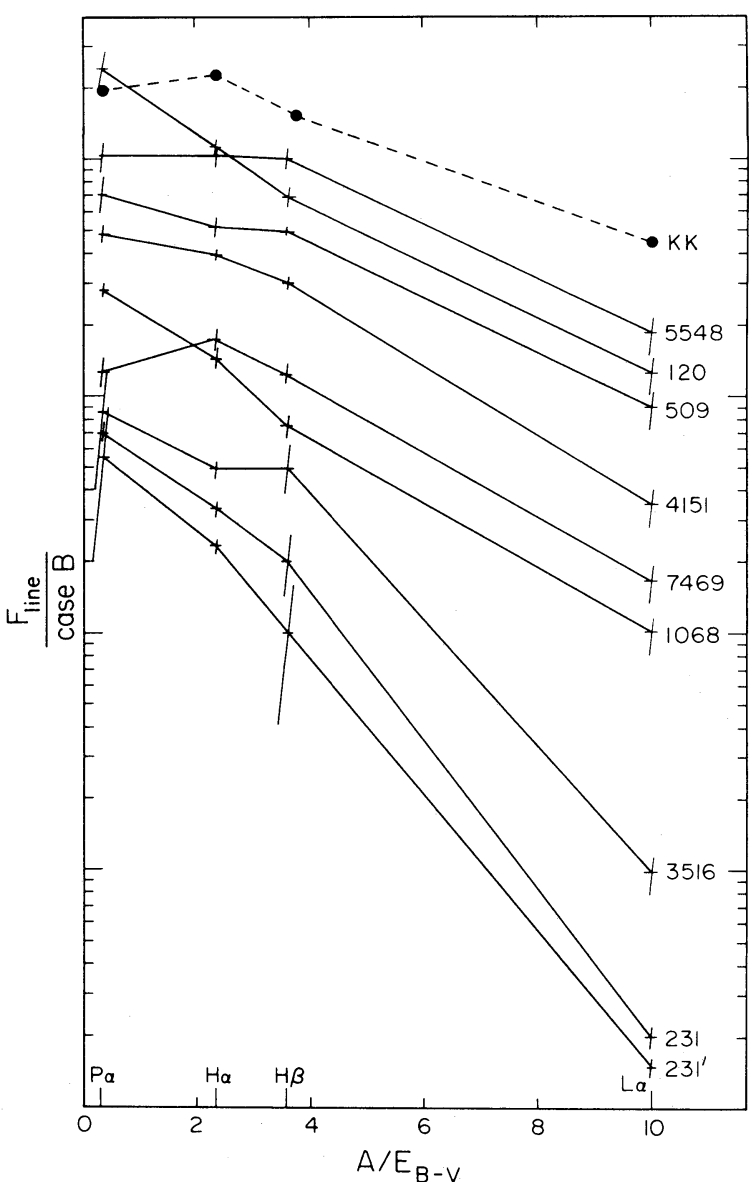

FIG. 3.-P $\alpha, H \alpha, H \beta$, and $L y \alpha$ line fluxes, each divided by the case $B$ ratio of that line to $\mathrm{H} \beta$, plotted on a logarithmic scale against $A / E_{B-\nu}$. Each galaxy has a different arbitrary normalization. Line fluxes from a unreddened $\mathrm{H}$ II region would fall on a horizontal line; reddening would result in a straight line with a negative slope proportional to $E_{B-V}$. The amount by which Ly $\alpha$ falls above or below a line through $\mathrm{P} \alpha$ and $\mathrm{H} \beta$ on this plot indicates the extent to which the dereddened $\mathrm{Ly} \alpha / \mathrm{H} \beta$ ratio, with the reddening derived from $\mathrm{P} \alpha / \mathrm{H} \beta$, differs from the adopted case B ratio of 35 . The line labeled $\mathrm{KK}$ is from the model of Kwan and Krolik (1981) with $N_{0}=4 \times 10^{9} \mathrm{~cm}^{-3}$.

\section{COMPARISON WITH MODELS}

Many models have been proposed to account for the deviations of hydrogen line ratios in Seyfert galaxies and quasars from the case B ratios. Most involve dense $\left(N \sim 10^{10} \mathrm{~cm}^{-3}\right)$ gas clouds photoionized by central nonthermal continuum sources. Calculations published prior to 1979 are reviewed by Davidson and Netzer (1979). In this section we first discuss the effects of large optical depths and high densities, then compare specific model calculations with the observed line fluxes, and finally discuss the evidence for reddening.

At high Lyman line optical depths where Ly $\alpha$ trapping maintains a significant population in the $n=2$ level of hydrogen atoms, Balmer and higher series transitions can become optically thick. At moderate optical depths, $\mathrm{H} \beta$ has a larger optical depth and smaller escape probability than does $\mathrm{P} \alpha$ so that trapped $\mathrm{H} \beta$ photons are converted into $\mathrm{P} \alpha+\mathrm{H} \alpha$. This effect can enhance $\mathrm{P} \alpha / \mathrm{H} \beta$ and $\mathrm{H} \alpha / \mathrm{H} \beta$ by factors of up to $\sim 3$, while increasing $\mathrm{P} \alpha / \mathrm{H} \alpha$ by a substantially smaller factor. At very large optical depths, $\mathrm{H} \alpha$ trapping populates $n=3$, allowing $\tau_{\mathrm{P} \alpha}>\tau_{\mathrm{H} \beta}$, and $\mathrm{P} \alpha+\mathrm{H} \alpha$ are converted back into $\mathbf{H} \beta$ (see Davidson and Netzer 1979). Conversions of $\mathrm{H} \beta$ to and from $\mathrm{P} \alpha+\mathrm{H} \alpha$ would result in ratios in Figure 2 to the right or left of the case B point, but would have little effect on $\mathrm{P} \alpha / \mathrm{H} \alpha$ and so could not explain the large variations observed in this ratio.

Collisional deexcitation of $n=2$ and excitation of $n=3$ would tend to increase $\mathrm{H} \alpha / \mathrm{Ly} \alpha$, as required by the observations. Collisional excitation of $n=4$ could decrease $\mathrm{P} \alpha / \mathrm{H} \beta$, since collisions tend to populate low angular momentum sublevels. However, the more rapid collisional transitions between sublevels would probably keep $\mathrm{P} \alpha / \mathrm{H} \beta$ near 0.28. Collisional enhancement of $\mathrm{H} \alpha$ at absorbing Ly $\alpha$ radiation, by a factor of $\sim 6$, than is external dust which produces the same amount of $\mathrm{P} \alpha / \mathrm{H} \beta$ reddening, because of the increased path traveled by a trapped Ly $\alpha$ photon before it can escape from a cloud (see, e.g., Davidson and Netzer 1979). Consequently, dust internal to an emission line cloud with an optical depth at Ly $\alpha$ of, e.g., $\tau_{\text {dust }}=0.3$ would decrease the Ly $\alpha$ flux by a factor of $\sim 6$ while absorbing little Lyman continuum and essentially no Balmer and Paschen line radiation. The galaxies whose $\mathrm{P} \alpha, \mathrm{H} \alpha$, and $\mathrm{H} \beta$ line fluxes lie along the reddening line in Figure 2 can be explained, within the uncertainties, by reddening by some combination of dust far from, near, or within the line-emitting regions. In most cases either external or internal dust alone would not be sufficient, since the amount of internal dust required to explain the $\mathrm{P} \alpha / \mathrm{H} \alpha / \mathrm{H} \beta$ ratios would absorb an unacceptably large fraction of the ionizing continuum, whereas external dust alone would not in general explain the observed $\mathrm{P} \alpha / \mathrm{H} \beta / \mathrm{Ly} \alpha$ ratios. Dust near the galactic nuclei might explain the observations if the albedo at Ly $\alpha$ is sufficiently less than that at $\mathrm{H} \alpha$ and $\mathrm{H} \beta$.

If the observed hydrogen line ratios are in fact substantially affected by reddening, the obscuring dust should have several other observable effects. Of the five Seyfert galaxies which appear most heavily reddened in Figure 3, NGC 1068, Mrk 231, Mrk 348, 3C 120, and 3C 227, four show other evidence of the presence of dust. As discussed by Neugebauer et al. (1980), NGC 1068 has He II and [S II] flux ratios consistent with $E_{B-V}=0.4$ mag, a steep infrared continuum probably due to emission from dust, and a $10 \mu \mathrm{m}$ absorption feature attributed to silicate grains. NGC 1068 does not, however, show the $2175 \AA$ absorption feature associated with reddening in the Galaxy. A steep infrared continuum and a large $\mathrm{P} \alpha / \mathrm{H} \beta$ flux ratio are also seen in the other Seyfert 2 observed, Mrk 348. Markarian 231 is unique among Seyfert 1 galaxies in its red color and the strong interstellar absorption lines in its spectrum. Allen et al. (1977) calculate $E_{B-V} \sim 0.6 \mathrm{mag}$ from the depths of the $\mathrm{Ca}$ II $\mathrm{H}$ and $\mathrm{K}$ lines, assuming a solar calcium abundance and normal gas-to-dust ratio. Markarian 231 also shows a $10 \mu \mathrm{m}$ silicate absorption feature. The ultraviolet 
spectrum of $3 \mathrm{C} 120$ has one of the strongest $2175 \AA$ features observed in an extragalactic object. Oke and Zimmerman (1979) estimate that it corresponds to a reddening of $E_{B-V}=0.38 \mathrm{mag}$. The presence of other evidence of reddening in those galaxies with the largest $\mathrm{P} \alpha / \mathrm{H} \beta$ ratios strengthens our conclusion that reddening is significant in at least some Seyfert galaxies.

The limits, which are discussed above, on the could help to explain the flux ratios of NGC 1275, NGC 7469, and MCG 8-11-11 which lie along the $\mathrm{P} \alpha / \mathrm{H} \beta=0.28$ line in Figure 1. Excitation of $n=3$ and deexcitation of $n=2$ both occur in regions of large Ly $\alpha$ optical depth where trapping of $\operatorname{Ly} \alpha$ maintains the $n=2$ population. Sufficient optical depths to make collisional transitions important can occur in partially ionized regions which have both neutral atoms and sufficient electrons to excite them (Krolik and McKee 1978).

Kwan and Krolik $(1979,1981)$ have calculated the emission line spectra of uniform-density clouds ionized and heated by power law continuum sources. In their models, most of the Balmer and Paschen line emission comes from a region which is partially ionized by the soft X-ray tail of the continuum. Tracks of three models with different gas densities, showing the variation of the $\mathrm{P} \alpha / \mathrm{H} \alpha / \mathrm{H} \beta$ ratios with Lyman edge optical depth, $\tau_{\mathrm{L}}$, are shown in Figure 2. Values of $\tau_{\mathrm{L}}$ of $10^{4}-10^{6}$ are required to give $\mathrm{Ly} \alpha / \mathrm{H} \beta \lesssim 20$ and so are probably most appropriate to Seyfert and quasar emission line clouds. Kwan and Krolik's $N_{0}=10^{9} \mathrm{~cm}^{-3}$ model passes well to the right of the case $\mathrm{B}$ point for appropriate values of $\tau_{\mathrm{L}}$. No Seyfert galaxies are seen in that region of Figure 2, although a few quasars are near there. Their higher density models pass near several of the data points, but fail to explain the large variations observed in $\mathbf{P} \alpha / \mathbf{H} \alpha$. None of their models predicts $\mathbf{P} \alpha / \mathbf{H} \alpha>0.13$.

Canfield and Puetter (1981) have made calculations similar to those of Kwan and Krolik (1981), with an improved treatment of the line escape probabilities, but assuming constant temperature through the clouds. Their $N_{0}=10^{10} \mathrm{~cm}^{-3}$ model is shown in Figure 3 . Although this model follows a substantially different path from Kwan and Krolik's models, similar $\mathrm{P} \alpha / \mathrm{H} \alpha / \mathrm{H} \beta$ ratios are found for $\tau_{\mathrm{L}}=10^{4}-10^{6}$ which gives $\mathrm{Ly} \alpha / \mathrm{H} \beta$ in the observed range. The different path followed by Canfield and Puetter's model results from the assumption of constant temperature; the models agree near the optical depth where the temperatures agree (Puetter, private communication). Except at very low optical depths, $\tau_{\mathrm{L}}<1$, their models, like Kwan and Krolik's, fail to explain the large values of $\mathrm{P} \alpha / \mathrm{H} \alpha$ observed from some galaxies.

The hydrogen line flux ratios at the optical depth at which $\mathrm{C}_{\mathrm{II}} / \mathrm{Ly} \alpha=1 / 30$ and $\operatorname{Ly} \alpha / \mathrm{H} \beta=10$ for $\mathrm{K}$ wan and Krolik's $N_{0}=4 \times 10^{9} \mathrm{~cm}^{-3}$ model are shown in Figure 3. The $\mathrm{Ly} \alpha / \mathrm{H} \beta$ ratio depends most strongly on optical depth, decreasing as $\tau_{\mathrm{L}}$ increases. Except for NGC 3516 and Mrk 231, with extremely weak Ly $\alpha$ fluxes, the observed $\mathrm{Ly} \alpha / \mathrm{H} \beta$ ratios can be reproduced by Kwan and Krolik's models with appropriate choices of $\tau_{\mathbf{L}}$, although the relative values of $\mathrm{P} \alpha, \mathrm{H} \alpha$, and $\mathrm{H} \beta$ are not in general reproduced simultaneously.

Several conclusions can be drawn from comparison of models with the observations. First, no models in which collisional excitation and deexcitation and radiative transfer are the most important means of altering the line flux ratios can explain the large values of $\mathrm{P} \alpha / \mathrm{H} \alpha$ observed in a significant fraction of the observed galaxies. Collisional excitation tends to decrease $\mathrm{P} \alpha / \mathrm{H} \alpha$, and large optical depths leave it nearly unchanged. In addition, the models proposed by Kwan and Krolik (1981) and by Canfield and Puetter (1981) fail to explain the very low $\mathrm{P} \alpha / \mathrm{H} \alpha$ ratios seen in some quasars and Seyfert galaxies. Although the various models follow significantly different paths on the $(\mathrm{P} \alpha / \mathrm{H} \alpha, \mathrm{H} \alpha / \mathrm{H} \beta)$-plot, all stay within a region with $\mathrm{P} \alpha / \mathrm{H} \alpha<0.13$ and $\mathrm{P} \alpha / \mathrm{H} \beta>$ 0.20 . These limits are also satisfied by models of Avrett (private communication), who uses an improved treatment of radiative transfer, and by most of the models of Drake and Ulrich (1980), who consider a very wide range of densities, although at very high temperatures, $T_{e}=20,000 \mathrm{~K}$, or densities, $N_{e}>10^{12} \mathrm{~cm}^{-3}$, Drake and Ulrich obtain $\mathrm{P} \alpha / \mathrm{H} \beta \sim 0.1$. None of the observed points fall significantly below $\mathrm{P} \alpha / \mathrm{H} \beta=0.20$, but several have $\mathrm{P} \alpha / \mathrm{H} \alpha>0.13$.

Reddening by dust offers a natural explanation for the large $\mathrm{P} \alpha / \mathrm{H} \alpha$ ratios observed. Although several authors (e.g., Osterbrock 1977) have shown that reddening cannot by itself explain the deviation of the hydrogen line spectra of Seyfert 1 galaxies from case B spectra, many of the galaxies in Figure 2 lie near the reddening track and, as discussed above, several have $\mathrm{P} \alpha / \mathrm{H} \alpha$ too large to be explain by any published model without reddening. In addition, at least two Seyfert galaxies have $\mathrm{P} \alpha, \mathbf{H} \alpha$, $\mathrm{H} \beta$, and $\mathrm{Ly} \alpha$ fluxes which all fall on reddening lines in Figure 3. Most of the other galaxies have $\mathrm{P} \alpha / \mathbf{H} \alpha / \mathbf{H} \beta$ ratios consistent with reddened case $B$ spectra, but have $\mathrm{Ly} \alpha / \mathbf{H} \beta$ suppressed by additional factors of up to 10 . The deviations of the line flux ratios from simple reddened case $\mathrm{B}$ ratios can be explained if either the emitted spectrum differs from a case B spectrum, or the reddening curve differs from the standard one. Both possibilities are considered below.

Since the densities and optical depths of the line emitting regions of Seyferts are thought to be too large for the case $B$ calculation to be applicable, variations from case B emitted spectra, like those discussed above, should probably be expected. At the optical depths which are thought to be relevant, Kwan and Krolik (1981) and Canfield and Puetter (1981) predict emitted $\mathrm{P} \alpha / \mathrm{H} \alpha / \mathrm{H} \beta$ ratios predominantly below and to the right of the case B point in Figure 2. Consequently, emitted spectra like those which they predict would, when reddened, match the observations better than would reddened case $B$ spectra. The various models also predict $\mathrm{Ly} \alpha / \mathrm{H} \beta<35$, again consistent with the dereddened observed ratios. Variations in the dereddened $\operatorname{Ly} \alpha / \mathrm{H} \beta$ ratio from galaxy to galaxy can be explained by variations in the optical thickness of the line emitting clouds.

The effect of dust on the hydrogen lines, especially Ly $\alpha$, could be altered if the dust is near or within the 
line-emitting region. Dust close enough to a galactic nucleus to be within the observed region $(\sim 0.5-5 \mathrm{kpc})$ would scatter light into as well as out of the line of sight. Since dust may have a higher albedo in the visible than in the ultraviolet (see Savage and Mathis 1979), Ly $\alpha$ could be more effectively attentuated relative to $\mathrm{H} \alpha$ and $\mathrm{H} \beta$ than expected with a standard extinction curve. In addition, dust mixed with the ionized gas is more effective $\mathrm{P} \alpha / \mathrm{H} \alpha / \mathrm{H} \beta$ ratios predicted by the models, $\mathrm{P} \alpha / \mathrm{H} \alpha<0.13$ and $\mathrm{P} \alpha / \mathrm{H} \beta>0.20$, can be used to limit the reddening required by each galaxy to a moderately narrow range. For those galaxies near the case B with reddening line, the differences among the models corresponds to an uncertainty in $E_{B-V}$ of $\pm 0.14 \mathrm{mag}$. The values of $E_{B-V}$ listed in Table 2, with this additional uncertainty, should represent the actual amount of reddening to these galaxies.

\section{CONCLUSIONS}

1. In many of the 18 observed Seyfert galaxies, $P \alpha$ is stronger relative to the Balmer lines than is predicted by recombination calculations.

2. In several Seyfert galaxies, as in most quasars observed by Soifer et al. (1981) and Puetter et al. (1981), $\mathrm{P} \alpha / \mathrm{H} \beta$ is near the recombination value, whereas $\mathrm{H} \alpha$ is enhanced relative to $\mathrm{P} \alpha$ and $\mathrm{H} \beta$. NGC 1275 is the most extreme object of this type.

3. The eight $\mathrm{Ly} \alpha / \mathrm{H} \beta$ ratios reported here are all less than expected from an $\mathrm{H}$ in region.

4. Reddening is the only mechanism which has been suggested that will enhance $\mathrm{P} \alpha$ relative to the Balmer lines by the amount observed in a significant number of the observed galaxies.

5. High densities and large optical depths are required in addition to reddening to explain the range of $\mathrm{P} \alpha / \mathrm{H} \alpha / \mathrm{H} \beta$ ratios. However, none of the proposed models can explain the flux ratios observed from NGC 1275.

6. Several effects can alter the $\mathrm{Ly} \alpha / \mathrm{H} \beta$ ratio. These include reddening, collisional excitation of $\mathrm{H} \beta$ and deexcitation of Ly $\alpha$, and large optical depths. Reddening by dust external to the galactic nuclei cannot by itself explain both the $\mathrm{P} \alpha / \mathrm{H} \beta$ and $\mathrm{Ly} \alpha / \mathrm{H} \beta$ ratios.

We thank W. L. W. Sargent, A. G. deBruyn, A. C. S. Readhead, and J. B. Oke for supplying optical spectra used in this paper and R. C. Puetter for helpful criticism. We are also grateful for the assistance of Juan Carrasco, Howard Lanning, Ron Koehler, Frank Cheigh, and the crew of the Kuiper Airborne Observatory.

This work was supported by grants from the NSF and NASA.

\section{REFERENCES}

Adams, T. F. 1977, Ap. J. Suppl., 33, 19.

Adams, T. F., and Weedman, D. W. 1975, Ap. J., 199, 19.

Allen, D. A., Boksenberg, A., Carswell, R. F., Fosbury, R. A. E.,

Penston, M. V., and Sargent, W. L. W. 1977, M.N.R.A.S., 178, 451.

Anderson, K. S. 1970, Ap. J., 162, 743.

Baldwin, J. A. 1977, Ap. J., 214, 679.

Boggess, A., et al. 1978a, Nature, 275, 372.

Boggess, A., et al. 1978b, Nature, 275, 377.

Bohlin, R., and Holm, A. V. 1980, IUE Newsletter, No. 10, p. 37

Canfield, R. C., and Puetter, R. C. 1981, Ap. J., 243, 390.

Davidson, A. F., Hartig, G., and Fastie, W. 1979, Nature, 269, 203.

Davidson, K., and Netzer, H. 1979, Rev. Mod. Phys., 51, 715.

Drake, S. A., and Ulrich, R. K. 1980, Ap. J. Suppl., 42, 351.

Grasdalen, G. L. 1976, Ap. J. (Letters), 208, L11.

Koski, A. T. 1978, Ap. J., 223, 56.

Krolik, J. H., and McKee, C. F. 1978, Ap. J. Suppl., 37, 459.
Kwan, J., and Krolik, J. H. 1979, Ap. J. (Letters), 233, L91.

1981, Ap. J., 250, 478.

Neugebauer, G., et al. 1980, Ap. J., 238, 502.

Oke, J. B., and Zimmerman, B. 1979, Ap. J. (Letters), 231, L13.

Osterbrock, D. E. 1974, Astrophysics of Gaseous Nebulae (San Francisco: Freeman).

- 1977, Ap. J., 215, 733.

Puetter, R. C., Smith, H. E., Willner, S. P., and Pipher, J. L. 1981, Ap. J., 243, 345.

Savage, B. D., and Mathis, J. S. 1979, Ann. Rev. Astr. Ap., 17, 73

Seaton, M. J. 1959, M.N.R.A.S., 119, 81 .

Soifer, B. T., Neugebauer, G., Oke, J. B., and Matthews, K. 1981, Ap. J., 243, 369.

Wampler, E. J. 1971, Ap. J., 164, 1.

Wu, C.-C., Boggess, A., and Gull, T. R. 1980, Ap. J., 242, 14.

E. E. Becklin: Institute for Astronomy, University of Hawaii, 2680 Woodlawn Drive, Honolulu, HI 96822

A. Boggess and T. R. Gull: Laboratory for Astronomy and Solar Physics, Goddard Space Flight Center, Greenbelt, MD 20771

J. H. Lacy, M. Malkan, K. Matthews, G. Neugebauer, and B. T. Soifer: Division of Physics, Mathematics, and Astronomy, Downs Laboratory 320-47, California Institute of Technology, Pasadena, CA 91125

Chi-Chao Wu: Astronomy Department, Computer Sciences Corporation, Goddard Space Flight Center, Greenbelt, MD 20771 